\title{
Lidil
}

Revue de linguistique et de didactique des langues

$52 \mid 2015$

Les pratiques artistiques dans l'apprentissage des langues

\section{Marion Tellier et Lucile Cadet (dir.), Le corps et la voix de l'enseignant : théorie et pratique}

Éditions Maison des Langues, 2014, $310 \mathrm{p}$.

\section{Federica Tummillo}

\section{OpenEdition \\ Journals}

Édition électronique

URL : http://journals.openedition.org/lidil/3907

DOI : 10.4000/lidil.3907

ISSN : $1960-6052$

\section{Éditeur}

UGA Éditions/Université Grenoble Alpes

\section{Édition imprimée}

Date de publication : 20 novembre 2015

Pagination : 206-208

ISBN : 978-2-84310-312-4

ISSN : $1146-6480$

\section{Référence électronique}

Federica Tummillo, «Marion Tellier et Lucile Cadet (dir.), Le corps et la voix de l'enseignant : théorie et pratique », Lidil [En ligne], 52 | 2015, mis en ligne le 01 janvier 2017, consulté le 29 octobre 2020. URL : http://journals.openedition.org/lidil/3907 ; DOI : https://doi.org/10.4000/lidil.3907 
Pour conclure, ce volume montre pourquoi il est capital de prendre en considération tout l'éventail des phénomènes communicatifs dans les recherches sur l'acquisition d'une langue seconde.

Federica Tummillo

GERCI, Université Grenoble Alpes

\begin{abstract}
Marion Tellier et Lucile Cadet (dir.), Le corps et la voix de l'enseignant : théorie et pratique, Éditions Maison des Langues, 2014, $310 \mathrm{p}$.
\end{abstract}

Les contributions réunies dans cette ouvrage prennent appui sur une constatation fondamentale : l'enseignant a un corps, et même une voix ! Une constatation qui a presque l'air d'une découverte, car «si les recherches sur le corps de l'enseignant en éducation et en didactique ont avant tout une coloration psychanalytique, sociologique $[\ldots] »$, expliquent Marion Tellier et Lucile Cadet, «celles qui portent sur les techniques de la voix et du corps (gestes, mimiques, postures) comme éléments pédagogiques, demeurent marginales». Le recueil réunit les travaux de chercheurs de disciplines différentes et se propose d'être une référence pour les enseignants, futurs, novices et expérimentés, afin de leur faire découvrir, développer et même protéger les outils naturels qu'ils emploient au quotidien dans leur métier. L'ouvrage s'adresse également aux chercheurs souhaitant s'initier à cette thématique ou l'approfondir.

Le recueil est divisé en trois parties, chacune composée d'une partie théorique suivie de fiches pratiques, visant pour la plupart à accompagner les enseignants dans la conscientisation, l'exploration et l'optimisation de leurs voix et leurs gestuelles. Des protocoles de recherche sont également présentés ainsi qu'un glossaire des concepts théoriques et techniques importants.

La première partie, La voix de l'enseignant, commence par l'introduction d'Élisabeth Guimbretière, phonéticienne et didacticienne des langues étrangères, qui donne des outils théoriques et pratiques pour comprendre les mécanismes physiologiques de la parole et la perception de la voix. La contribution de Camille Robieux et Antoine Giovanni aborde la question des «Troubles de la voix chez l'enseignant» 
dans une perspective médicale et orthophonique, tandis que, dans «La voix de l'enseignant : risques et prévention», Margaret Bento aborde la même problématique du point de vue de la formation. Ensuite Claire Pillot-Loiseau se penche sur le ressenti des enseignants de langue visà-vis de leur voix dans «Geste vocal, voix et enseignement des langues». Enfin, Malak Moustapha-Sabeur et José Aguilar Río étudient le discours de différents enseignants sur la perception et l'usage de leur voix en classe dans «Faire corps avec sa voix : paroles d'enseignants».

La deuxième partie traite du Corps de l'enseignant dans la classe, dans sa dimension posturo-mimo-gestuelle. L'introduction de Marion Tellier, gestualiste et didacticienne des langues étrangères, présente le premier état de l'art visant, entre autres, à définir les différentes dimensions des gestes en classe de langue et à démêler l'ambigüité entre certaines notions, comme celles de «gestualité coverbale» et «communication non verbale». Dans l'article, «Multimodalité des signes et enjeux énonciatifs en classe de FL1/FLS », Brahim Azaoui observe et analyse des situations de classe en focalisant l'attention sur ce qu'il définit «ubiquité coénonciative» dans la gestuelle de l'enseignant. Puis Marion Tellier, Laetitia Michel et Ludivine Wolff montrent que la gestuelle pédagogique varie en fonction des enseignants mais également des fonctions pédagogiques qu'elle sert dans «Variations inter et intra-individuelles de la gestuelle chez l'enseignante de maternelle». Ensuite, l'article «Fonctionnalité des gestes pour l'enseignement de la phonoprosodie», de Nadine Herry-Bénit et Lucile Cadet, montre comment la gestuelle peut être utilisée pour transmettre des éléments phonétiques et prosodiques dans l'enseignement d'une langue étrangère. Enfin, Caroline Scheepers et Alain Hertay examinent comment les enfants perçoivent le corps de leurs enseignants dans "Quand des enfants jouent à faire la classe : des hexis corporelles contrastées».

La troisième partie, Expérimentations et dispositifs de formation des enseignants, traite de la façon dont on peut aborder en formation les questions concernant la voix et le corps de l'enseignant. L'introduction de Lucile Cadet, didacticienne de langues étrangères, traite la façon dont recherche et formation abordent cette problématique. Dans «Conscientisation de la gestuelle pédagogique en formation initiale», Alexandra Gadoni et Marion Tellier présentent un dispositif d'autoconfrontation pour travailler sur le corps des futurs enseignants en formation. Ensuite Hervé Girault, dans «Enseignants en formation et formateurs face au conseil vocal», montre l'importance de la pratique du chant en formation tandis qu'Isabelle Jourdan, dans «Corps en scène 
et voix de l'affect», expose une démarche clinique de formation mettant en scène le corps et la voix dans diverses situations. Puis Valérie Lemeunier, Hugues Denisot et Raphaël Fernandez, dans «Concevoir des modules de formation pour faire du corps de l'enseignant un outil pédagogique», font connaitre différents modules de formation continue élaborés à partir d'activités de dynamique de groupe et de techniques théâtrales. Enfin, Yves Cusset, dans «À son corps défendant ou comment l'enseignant apprend à jouer son rôle», présente une expérience de formation à travers les pratiques théâtrales adaptée à la formation initiale d'enseignants du supérieur.

Pour conclure, on peut dire que s'il est vrai que l'évolution des méthodologies soulève de plus en plus la question de la participation du corps de l'apprenant dans le processus d'apprentissage (à partir des approches communicatives et de la perspective actionnelle jusqu'à l'intégration des pratiques artistiques en classe de langue), cet ouvrage montre que le corps de l'apprenant entre forcément en résonance avec celui de l'enseignant. D'ici la nécessité de sensibiliser les enseignants à cette thématique et de développer ultérieurement les recherches dans cette direction.

Federica Tummillo GERCI, Université Grenoble Alpes

Études romanes de Brno, volume 1 ( $n^{\circ}$ 35.1) : Sens multiple(s) et polysémie, regards d'Occident (dossier thématique coordonné par Julie Sorba et Christophe Cusimano); volume 2 ( $\left.n^{\circ} 35.2\right)$ : Sens multiple(s) et polysémie, regards d'Orient (dossier thématique coordonné par Julie Sorba et Sylvain Brocquet), 2014.

Le dossier présenté ci-dessous interroge, en deux phases, le phénomène «polysémie», théoriquement et dans ses usages, en Occident et dans l'Orient ancien.

\section{Volume 1. - Sens multiple(s) et polysémie, regards d'Occident}

F. Douay ouvre, sous les apparences d'une perspective historique, le débat : à forme unique, acceptions plurielles, et signale l'avancée notable de Fontanier qui, décrivant un sens par extension, entre sens 